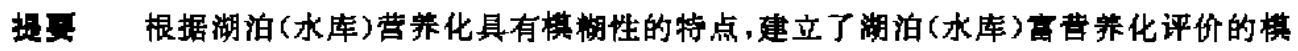
“蝴集理论与棋式,并首次提出畐苦美化指标综合权重矩阵概念及其确定方法。应用该棋式对我

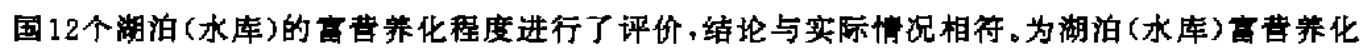
治理和保护提供了科学依据。上述理论与棋型原则上亦可在其他环境评价领域中应用。

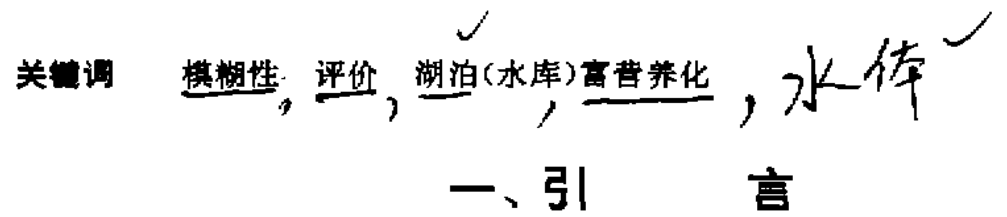

湖泊与水库 (以下简称为湖库) 中水体的富营养化,使水质恶化,不仅破坏湖库的游紧累 观，且严重影响沿湖库城市的工业和生活用水。例如1982年8月大伙房水库一次暴发性藻类， 生物量达到 $154 \mathrm{~g} / \mathrm{m}^{3}$, 使取水厂过滤器堵塞, 造成抚顺市供水紧张 (1) 又如 1990 年 7 月太湖的

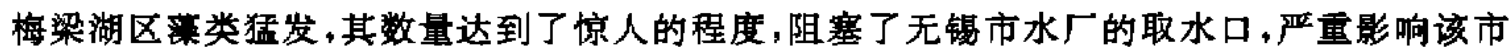
的生产和生活用水 ${ }^{2}$ 。因此,对于湖库水体富营养化的控制与治理，应引起足敌的重规。它是 湖库环境保护中一项极其重要的内容,对湖库水体的宫营养化评价则是控制与治理的基础。

为了准确评价湖库所处的营养状态, 进而为湖库富营养化的防治提供科学依据, 近半个 世纪以来，世界各国一些著名学者先后提出了特征法、参数法、营养状态指数法、生物评价法 等多种评价方法,在国外一些湖库富营养化评价的应用中收到比较好的效果。但是由于湖库 所处的地理位置、环境条件、人类活动影响、湖库富营养化的类型等不尽相同,其评价方法也 不完全一致。为了探讨适合我国主要是浮游植物型湖库富营养化程度的评价方法,文 [1]根 据国内1987-1988年14个有代表性湖库富营养化调查资料的综合分析,对我国湖库富营养 化程度综合评价的参数、标准和模式等作了深入的分析与探讨,提出捯选用国外应用较为广 泛的营养状态指数公式和应用简便的评分公式,作为我国湖库富营养化程度评价的基本模 式。

湖库宫营养化程度的轻重,并不具有明确的界限，存在着中介过渡性，即模糊性。因此，

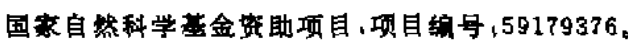

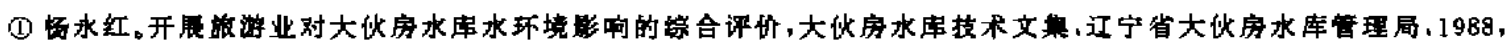
233-244。

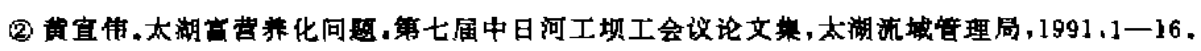

本文于1992年2月26日收到.1992年4月2日改回. 
在文 $[2,3]$ 的基础上,本文提出湖库富营养化模楜集评价理论与模式,以适合于湖库富营养 化评价之用。

\section{二、湖库富营莱化指标综合权重矩阵}

设有 $n$ 个湖库(或测点)水体营养化样本, 每个样本有 $m$ 项营养化评价指标的实测值。 苦养化程度的分级数为 $c$, 评价指标的标准浓度值为 $y$ 。则有 $c$ 级莒养化标准浓度矩阵(1) 与 湖库营养化实测浓度矩阵(2)。

$$
\begin{gathered}
Y_{m \times c}=\left[\begin{array}{llll}
y_{11} & y_{12} & \cdots & y_{1 c} \\
y_{21} & y_{22} & \cdots & y_{2 c} \\
\cdots & \cdots & \cdots & \cdots \\
y_{m 1} & y_{m 2} & \cdots & y_{m c}
\end{array}\right]=\left(y_{i h}\right) \\
X_{m \times n}=\left[\begin{array}{llll}
x_{11} & x_{12} & \cdots & x_{1 n} \\
x_{21} & x_{22} & \cdots & x_{2 n} \\
\cdots & \cdots & \cdots & \cdots \\
x_{m 1} & x_{m 2} & \cdots & x_{m n n}
\end{array}\right]=\left(x_{i j}\right) \\
i=1,2, \cdots, m, h=1,2, \cdots, c ; j=1,2, \cdots, n 。
\end{gathered}
$$

考虑湖库水体“营养化”是模糊概念, 可用模楜集合中的隶属度来描述。本文规定 1 级营

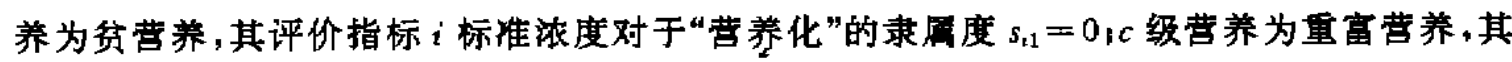
评价指标 $i$ 的标准浓度对“营养化”的隶風度 $s_{\mathrm{ac}}=1$ 。则湖库水体 $h$ 级营养评价指标 $i$ 标准浓 度的隶属度 $s_{i h}$ 在 $[0,1]$ 区间, 可按线性公式(3)确定。

$$
s_{t h}=\frac{y_{t h}-y_{i 1}}{y_{t c}-y_{i 1}}
$$

式中 $y_{11} 、 y_{i h} 、 y_{i c}$ 分别为 1 级、 $h$ 级、 $c$ 级营养化评价指标 $i$ 的标准浓度。由式 (3) 可将矩阵 (1) 变 换为湖库水体营美化标准模糊矩阵:

$$
\underline{\sim}_{m \times c}=\left[\begin{array}{cccc}
s_{11} & s_{12} & \cdots & s_{1 c} \\
s_{21} & s_{22} & \cdots & s_{2 \mathrm{c}} \\
\cdots & \cdots & \cdots & \cdots \\
s_{m 1} & s_{m 2} & \cdots & s_{m c}
\end{array}\right]=\left(s_{t h}\right)
$$

类似地，可根据式 $(5 a)$

$$
r_{1 j}= \begin{cases}1, & x_{i j}>y_{t c} \\ \frac{x_{i,}-y_{i 1}}{y_{i c}-y_{i 1}}, & y_{i 1} \leqslant x_{i j} \leqslant y_{i c} \\ 0, & x_{i j}<y_{i 1}\end{cases}
$$

将透明度除外的营美化评价指标实测浓度变换为实测浓度模糊矩阵中对应元亲的隶風度 $r_{1,0}$

对于透明度因其数值越小,湖库营养化程度越重,故用下式进行变换。 


$$
r_{i s}= \begin{cases}1, & x_{i j}<y_{i c} \\ \frac{x_{i 1}-y_{11}}{y_{i 1}-y_{i c}}, & y_{i c} \leqslant x_{1 j} \leqslant y_{d 1} \\ 0, & x_{i j}<y_{i 1}\end{cases}
$$

由式 $(5 a) 、(5 b)$ 可将矩阵 (2) 变换为湖库营苏化评价指标实测浓度模糊矩阵:

$$
\underset{\sim \times n}{\boldsymbol{R}_{m \times n}}=\left[\begin{array}{cccc}
r_{11} & r_{12} & \cdots & r_{1 n} \\
r_{21} & r_{22} & \cdots & r_{2 n} \\
\cdots & \cdots & \cdots & \cdots \\
r_{m 1} & r_{m 2} & \cdots & r_{m n}
\end{array}\right]=\left(r_{13}\right)
$$

矩阵 (6) 有著明确的物理意义，它表达了 $n$ 个湖库(或测点) $m$ 个指标实测浓度对于模糊概念 “营苏化”的作用大小或影响程度。它的数学意义是描述了全体样本全部指标对于模糊子集 “营羊化”的隶局度。根据模糊集合论可将隶局度定义为权重的论点 ${ }^{[4]}$, 故可取营苏化指标实 测浓度模糊矩阵 (6)作为样本集在不同指标浓度情况下的权重矩阵。它给出了样本集的各个

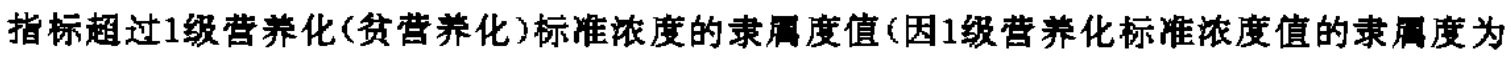
0)。但隶禹度不仅是表示了超标值，而且是权重，故矩阵(6)也称超标权重矩阵。

此外， $m$ 项营养化评价指标对富营养化的影响常常并不完全相同。例如湖库中磷、氮对 富营养化影响比透明度更大。故还应考思 $m$ 项评价指标本身对富营前化作用大小的权重， 故称为指标权重,可用指标权向量表示为

$$
\vec{v}=\left(v_{1}, v_{2}, \cdots, v_{m}\right), \quad \sum_{i=1}^{m} v_{i}=1
$$

综合考㤐超标权重与指标权重,可得 $n$ 个样本 $m$ 项评价指标的综合权重模式

$$
\begin{aligned}
{\underset{\sim}{m \times n}}_{m} & =\left[\begin{array}{cccc}
v_{1} & 0 & \cdots & 0 \\
0 & v_{2} & \cdots & 0 \\
\cdots & \cdots & \cdots & \cdots \\
0 & 0 & \cdots & v_{m}
\end{array}\right] \cdot\left[\begin{array}{cccc}
r_{11} & r_{12} & \cdots & r_{1 n} \\
r_{21} & r_{22} & \cdots & r_{2 n} \\
\cdots & \cdots & \cdots & \cdots \\
r_{m 1} & r_{m 2} & \cdots & r_{m n}
\end{array}\right] \\
& =\left[\begin{array}{cccc}
v_{1} r_{11} & v_{1} r_{12} & \cdots & v_{1} r_{1 n} \\
v_{2} r_{21} & v_{2} r_{22} & \cdots & v_{2} r_{2 n} \\
\cdots & \cdots & \cdots & \cdots \\
v_{m} r_{m 1} & v_{m} r_{m 2} & \cdots & v_{m} r_{m n}
\end{array}\right]=\left(v_{i} r_{i j}\right)
\end{aligned}
$$

将矩阵 $(8)$ 元亲值按列归一化, 得到

$$
\underset{m \times n}{\underset{W}{W}}=\left[\begin{array}{cccc}
w_{11} & w_{12} & \cdots & w_{1 n} \\
w_{21} & w_{22} & \cdots & w_{2 n} \\
\cdots & \cdots & \cdots & \cdots \\
w_{m 1} & w_{m 2} & \cdots & w_{m n}
\end{array}\right]=\left(w_{1 j}\right)
$$

式中 $w_{i}$ 为样本 $j$ 第 $i$ 个指标的综合权重,其表达式为

$$
w_{i j}=\frac{v_{i} r_{i j}}{\sum_{i=1}^{m} v_{i} r_{i j}}, \quad \sum_{i=1}^{m} w_{i j}=1
$$


本文称矩阵 (9)为指标的综合权重矩阵,它表示了样本集各个指标对湖库“营养化”作用不 同，而应赋予的不同权重。对“营养化”作用大的指标,其综合权重也大。

上面提出的指标综合权重矩阵及确定方法, 突破了现代文献中关于环境评价指标权重 确定的传统观点与方法.具有理论与实际意义。

\section{三、湖库营养化模糊最优分级模式}

设湖库(或测点)的第 $j$ 个样本用向量表示为

$$
\vec{r},=\left(r_{1 s}, r_{2}, \cdots, r_{m j}\right)^{T}
$$

而湖库营养化第 $h$ 级的评价指标标准为

$$
\overrightarrow{s_{h}}=\left(s_{1 h}, s_{2 h}, \cdots, s_{m h}\right)^{T}
$$

样本 $j$ 的指标权向量为

$$
\vec{w}_{j}=\left(w_{1 s}, w_{2 s}, \cdots, w_{m p}\right)^{T}
$$

则样本 $j$ 与第 $h$ 级湖库营养化指标标准之间的差异可用者虑样本 $j$ 指标权向量的欧氏加权 距离式(11)表示。

$$
\left\|\vec{w},\left(\vec{r}_{,}-\vec{s}_{h}\right)\right\|=\sqrt{\sum_{i=1}^{m}\left[w_{1},\left(r_{i}-s_{i h}\right)\right]^{2}}
$$

根据湖库营养化分级的模糊性, 样本以不同的隶属度 $u$ 隶属于各级营养化标准。设 $n$ 个 样本对 $c$ 级湖库营养化标准的隶属度以模糊矩阵

表示。其约束条件为

$$
\underset{\sim}{U_{c \times n}}=\left[\begin{array}{cccc}
u_{11} & u_{12} & \cdots & u_{1 n} \\
u_{21} & u_{22} & \cdots & u_{2 n} \\
\cdots & \cdots & \cdots & \cdots \\
u_{c 1} & u_{c 2} & \cdots & u_{c n}
\end{array}\right]=\left(u_{h j}\right)
$$

$$
\sum_{h=1}^{c} u_{h_{j}}-1=0, \quad \sum_{j=1}^{n} u_{h j}>0
$$

为求解矩阵 (12) 的最优分级矩阵, 可建立目标函数：

$$
\begin{gathered}
\min \left\{F\left(u_{h j}\right)\right\}=\min \left\{\sum_{j=1}^{n} \sum_{k=1}^{c}\left[u_{k j} \sqrt{\left.\sum_{i=1}^{m}\left[w_{i},\left(r_{i},-s_{1 k}\right)\right]^{2}\right]^{2}}\right\}\right. \\
=\sum_{j=1}^{n} \min \left\{\sum_{k=1}^{c} u_{h,}^{2}\left[\sum_{i=1}^{m}\left[w_{i},\left(r_{1},-s_{t h}\right)\right]^{2}\right]\right\}
\end{gathered}
$$

构造拉格朗日函数:

$$
L\left(u_{k j}, \lambda\right)=\sum_{h=1}^{c} u_{i j}^{2}\left[\sum_{i=1}^{m}\left[w_{1,}\left(r_{t s}-s_{i k}\right)\right]^{2}\right]-\lambda\left(\sum_{h=1}^{c} u_{1 j}-1\right)
$$

分别对拉格朗日乘数 $\lambda$ 与 $u_{k j}$ 求函数 $L\left(u_{h s}, \lambda\right)$ 的偏导数, 且令偏导数等于零, 得

$$
\frac{\partial L\left(u_{h,}, \lambda\right)}{\partial \lambda}=\sum_{h=1}^{c} u_{h j}-1=0
$$




$$
\frac{\partial L\left(u_{h,}, \lambda\right)}{\partial t_{h \jmath}}=2 u_{h j}\left[\sum_{i=1}^{m}\left[w w_{1,}\left(r_{i,}-s_{i h}\right)\right]^{2}\right]-\lambda=0
$$

由式 (16)、(17)解得最优分级矩阵元紊的表达模式为

$$
U_{h s}=\frac{1}{\sum_{k=1}^{c} \frac{\sum_{i=1}^{m}\left[w_{1,}\left(r_{1,}-s_{t h}\right)\right]^{2}}{\sum_{i=1}^{m}\left[w_{i j}\left(r_{1,}-s_{t k}\right)\right]^{2}}}
$$

本文称式 (18) 为湖库营养化模糊最优分级模式。根据它可以计算确定最优分级矩阵的每一 个元东，从而得到样本集对于各级营养化的隶属度，据此可对湖库(或测点)的富营养化程度 作出评价。

\section{四、我国湖库富营美化评价指标权重的确定}

我国湖库主要是浮游植物型,其营养化现象的发生主要是湖库中磷、等营养物质增 加，导致落类过量繁殖所致。一般均以反映湖库藻类数量多骞的综合指标: 叶绿紊 a 作为占 主导地位的评价指标。文[1]统计分析了我国 14个有代表性湖库水质、生物资料,得到 TP $\left(\mathrm{mg} / \mathrm{m}^{3}\right) 、 \mathrm{TN}\left(\mathrm{mg} / \mathrm{m}^{3}\right) 、 C O D_{M n}(\mathrm{mg} / \mathrm{L}) 、 \mathrm{SD}(\mathrm{m})$ 与主导指标 Chl-a $\left(\mathrm{mg} / \mathrm{m}^{3}\right)$ 的相关关系，它 们与 $\mathrm{Chl}-\mathrm{a}$ 相关系数较大,故建议采用上述指标为我国湖库富营养化评价基本指标。

由于叶绿京 a 是湖库富营养化主导评价指标,因叶绿京 $\mathrm{a}$ 与本身的关系最密切,其相关 系数为1。显然, 与叶绿素 a 相关性 (以相关系数表示) 大的指标, 其权重也应大。即指标权重 应与该指标同叶绿亲 $\mathrm{a}$ 的相关系数绝对值成正比。设 $\mathrm{m}$ 个评价指标中 Chl-a 为第1个指标， 其余指标与 Chl-a 的相关系数分别为 $r_{2}, r_{3}, \cdots, r_{m}$ 。因此可以取评价指标与 Chl-a 的相关系 数的绝对值为 $m$ 个指标末经归一化的权向量:

$$
\vec{v}^{\prime}=\left(\left|r_{1}\right|,\left|r_{2}\right|, \cdots,\left|r_{m}\right|\right)
$$

归一化得指标权向量：

$$
\begin{aligned}
\vec{v} & =\left(\frac{\left|r_{1}\right|}{\sum_{i=1}^{m}\left|r_{1}\right|}, \frac{\left|r_{2}\right|}{\sum_{i=1}^{m}\left|r_{1}\right|}, \cdots, \frac{\left|r_{m}\right|}{\sum_{i=1}^{m}\left|r_{1}\right|}\right) \\
& =\left(v_{1}, v_{2}, \cdots, v_{m}\right)
\end{aligned}
$$

式中 $r_{1}$ 为 Chl-a 与本身的相关系数, $r_{1}=1$ 。

\section{五、应用实 例}

在湖库萝类䇣殖的高峰期, 我国 12 个湖库 Chl-a、TP、TN、COD、SD 评价指标的实测浓 度资料如表 1 所列 $[1]$ 。试根据本文提出的模式，对12个湖库的营养化程度进行综合评价。

我国湖库营养化评价标准文[1]进行了仔细的分析研究工作,提出的评价标准见表2。 
表1 我国12个湖库评价指标的实湖浓度值

Tab. 1 The testing data of assessment indices for the 12 lakes (reservoirs) in China

\begin{tabular}{|c|c|c|c|c|c|c|}
\hline 序号 & 湖库名称 & $\begin{array}{c}\text { Chl-a } \\
\left(m g / m^{3}\right)\end{array}$ & $T P\left(m g / m^{3}\right)$ & $\begin{array}{c}T N \\
\left(m g / m^{3}\right)\end{array}$ & $\underset{\{\mathrm{mg} / \mathrm{L}\}}{\mathrm{COD}}$ & $\begin{array}{l}\text { SD } \\
(m)\end{array}$ \\
\hline 1 & IS & 0.88 & 130 & 410 & 1.43 & 2.98 \\
\hline 2 & 海 & 4. 33 & 21 & 180 & 3. 38 & 2. 40 \\
\hline 3 & 博斯沟湖 & 4. 91 & 50 & 969 & 5. 42 & 1.46 \\
\hline 4 & 于桥水库 & 16.20 & 26 & 1020 & 5. 16 & 1.16 \\
\hline 5 & 玩 & 15.38 & $B ?$ & 1540 & 4.40 & 0.65 \\
\hline 6 & 雀 & 14.56 & 140 & 2270 & 4.34 & 0.27 \\
\hline$?$ & 甘萧 湖 & 73.70 & 135 & 2140 & 6. 96 & 0.36 \\
\hline 8 & 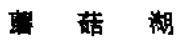 & 82.40 & 332 & 2660 & 14. 60 & 0.49 \\
\hline 9 & 杭州 西 湖 & 95.94 & 136 & 2230 & 10.18 & 0.37 \\
\hline 10 & 南京玄武湖 & 202.10 & 708 & 6790 & B. 86 & 0.31 \\
\hline 11 & 武没悬水湖 & 262.40 & 500 & 16050 & 13.60 & 0.15 \\
\hline 12 & 广州东山湖 & 185.10 & 670 & 7200 & 14.80 & 0.26 \\
\hline
\end{tabular}

表2 我国湖库营养化评价标准

Tab. 2 The assessment standards of the lake (reservoir) eutrophication

\begin{tabular}{|c|c|c|c|c|c|c|c|}
\hline 序号 & 评仆指标 & 委营来 & 穹中書养 & 中营荠 & 中宮苦券 & 番苦莱 & 重畐营亜 \\
\hline 1 & $\underset{\left(\mathrm{mg} / \mathrm{m}^{3}\right)}{\mathrm{Chl}}$ & 1.0 & 2.0 & 4.0 & 10 & 65 & 160 \\
\hline 2 & $\begin{array}{c}\mathrm{TP} \\
\left(\mathrm{mg}_{\mathrm{g}} / \mathrm{m}^{3}\right)\end{array}$ & 2.5 & 5.0 & 25 & 50 & 200 & 600 \\
\hline 3 & $\begin{array}{c}\text { TN } \\
\left(\mathrm{mg}_{\mathrm{g}} / \mathrm{m}^{3}\right)\end{array}$ & 30 & 50 & 300 & 500 & 2000 & 6000 \\
\hline 4 & $\underset{(\mathrm{mg} / \mathrm{L})}{\mathrm{COD}}$ & 0.3 & 0.4 & 2.0 & 4.0 & 10 & 25 \\
\hline 5 & $\begin{array}{l}\mathrm{SD} \\
(\mathrm{m})\end{array}$ & 10.0 & 5.0 & 1.5 & 1.0 & 0.4 & 0.3 \\
\hline
\end{tabular}

根据表 $1 、 2$ 有

$$
X_{5 \times 12}=\left[\begin{array}{cccccccccccc}
0.88 & 4.33 & 4.91 & 16.20 & 15.38 & 14.56 & 73.30 & 82.40 & 95.94 & 202.10 & 262.40 & 185.107 \\
130 & 21 & 50 & 26 & 87 & 140 & 135 & 332 & 136 & 308 & 500 & 630 \\
410 & 180 & 969 & 1020 & 1540 & 2270 & 2140 & 2660 & 2230 & 6790 & 16050 & 7200 \\
1.43 & 3.38 & 5.42 & 5.16 & 4.40 & 4.34 & 6.96 & 14.60 & 10.18 & 8.86 & 13.60 & 14.80 \\
2.98 & 2.40 & 1.46 & 1.16 & 0.65 & 0.27 & 0.36 & 0.49 & 0.37 & 0.31 & 0.15 & 0.26
\end{array}\right]
$$$$
Y_{5 \times 1}=\left[\begin{array}{cccccc}
1.0 & 2.0 & 4.0 & 10 & 65 & 160 \\
2.5 & 5.0 & 25 & 50 & 200 & 600 \\
30 & 50 & 300 & 500 & 2000 & 6000 \\
0.3 & 0.4 & 2.0 & 4.0 & 10 & 25 \\
10.0 & 5.0 & 1.5 & 1.0 & 0.4 & 0.3
\end{array}\right]=\left(y_{\text {in }}\right)
$$

$$
i=1,2, \cdots, 5 ; j=1,2, \cdots, 12 ; h=1,2, \cdots, 6
$$

由式 (3) 将矩阵 $\left(1^{\prime}\right)$ 转换为营养化标准模楜矩阵： 


$$
\underset{5 \times 3}{S_{5 \times 3}}=\left[\begin{array}{llllll}
0 & 0.006 & 0.019 & 0.057 & 0.402 & 1 \\
0 & 0.004 & 0.038 & 0.080 & 0.330 & 1 \\
0 & 0.003 & 0.045 & 0.079 & 0.330 & 1 \\
0 & 0.004 & 0.069 & 0.150 & 0.393 & 1 \\
0 & 0.515 & 0.876 & 0.928 & 0.989 & 1
\end{array}\right]=\left(S_{1 h}\right)
$$

对透明度用式 $(5 b)$, 其余指标用 (5a) 将矩阵 $\left(2^{\prime}\right)$ 转换为指标实测浓度模楜矩阵 $\left(6^{\prime}\right)$ 。

$$
\underset{\sim}{R_{512}}=\left[\begin{array}{cccccccccccc}
0 & 0.021 & 0.025 & 0.096 & 0.090 & 0.085 & 0.482 & 0.512 & 0.547 & 1 & 1 & 1 \\
0.213 & 0.031 & 0.079 & 0.039 & 0.141 & 0.230 & 0.222 & 0.551 & 0.223 & 1 & 0.833 & 1 \\
0.064 & 0.025 & 0.157 & 0.166 & 0.253 & 0.375 & 0.353 & 0.441 & 0.369 & 1 & 1 & 1 \\
0.046 & 0.125 & 0.207 & 0.147 & 0.166 & 0.164 & 0.270 & 0.579 & 0.400 & 0.347 & 0.538 & 0.587 \\
0.724 & 0.784 & 0.880 & 0.911 & 0.964 & 1 & 0.994 & 0.980 & 0.993 & 0.994 & 1 & 1
\end{array}\right]=\left(r_{13}\right) \quad\left(6^{\prime}\right)
$$

文[1]统计分析了我国14个有代表性湖库的 Chla 与 TP、TN、COD、SD 的相关系数,分 别为 $0.93 、 0.81 、 0.90 、-0.65$, 于是有 $\left|r_{2}\right|=0.93 、\left|r_{3}\right|=0.81,\left|r_{4}\right|=0.90,\left|r_{5}\right|=0.65$ 。 因 $\left|r_{1}\right|=1$ ，根据式(19)有

$$
\overrightarrow{v^{r}}=(1,0.93,0.81,0.90,0.65)
$$

将式 $\left(19^{\prime}\right)$ 归一化,得指标权向量

$$
\vec{v}=\left(v_{1}, v_{2}, v_{3}, v_{4}, v_{5}\right)=(0.233,0.217,0.189,0.210,0.151)
$$

由综合权重模式 ( 8 ), 得

$$
A_{5 \times 12}=\left[\begin{array}{cccccccccccc}
0 & 0.005 & 0.006 & 0.022 & 0.021 & 0.020 & 0.112 & 0.119 & 0.139 & 0.233 & 0.233 & 0.233 \\
0.046 & 0.007 & 0.017 & 0.008 & 0.031 & 0.050 & 0.048 & 0.120 & 0.048 & 0.217 & 0.181 & 0.217 \\
0.012 & 0.005 & 0.030 & 0.031 & 0.048 & 0.071 & 0.067 & 0.083 & 0.070 & 0.189 & 0.189 & 0.189 \\
0.010 & 0.026 & 0.043 & 0.041 & 0.035 & 0.034 & 0.057 & 0.122 & 0.084 & 0.073 & 0.113 & 0.123 \\
0.109 & 0.118 & 0.133 & 0.138 & 0.146 & 0.151 & 0.150 & 0.148 & 0.150 & 0.151 & 0.151 & 0.151
\end{array}\right]=\left\{v_{1} r_{0}\right\rangle
$$

由式 (10) 将矩阵 $\left(8^{r}\right)$ 按列归一化, 得指标综合权重矩阵:

\begin{tabular}{|c|c|c|c|c|c|c|c|c|c|c|c|c|c|}
\hline ( & 【1) & (2) & (3) & (4) & (5) & (6) & (7) & (8) & (9) & $(10)$ & (11) & (12) & (级别) \\
\hline \multirow{6}{*}{$f_{6 \times 12}=$} & 0.019 & 0.008 & 0.002 & 0.001 & 0.002 & 0.004 & 0.005 & 0.027 & 0.018 & 0.013 & 0.022 & 0.0147 & 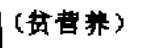 \\
\hline & 0.202 & 0.065 & 0.009 & 0.004 & 0.011 & 0.016 & 0.016 & 0.051 & 0.041 & 0.014 & 0.025 & 0.016 & 晏菜) \\
\hline & 0.361 & 0.544 & 0.471 & 0.184 & 0.175 & 0.098 & 0.038 & 0.078 & 0.069 & 0.016 & 0.028 & 0.017 & 莱》 \\
\hline & 0.230 & 0.228 & 0.439 & 0.751 & 0.495 & 0.158 & 0.049 & 0.098 & 0.084 & 0.017 & 0.030 & 0.019 & 宫乐） \\
\hline & 0.138 & 0.102 & 0.069 & 0.050 & 0.301 & 0.703 & 0.875 & 0.658 & 0.727 & 0.036 & 0.068 & 0.041 & 莱) \\
\hline & .050 & 0.053 & 0.010 & 0.005 & 0.016 & 0.021 & 0.017 & 0.088 & 0.061 & 0.904 & 0.827 & 0.893 & \{营养\} \\
\hline
\end{tabular}

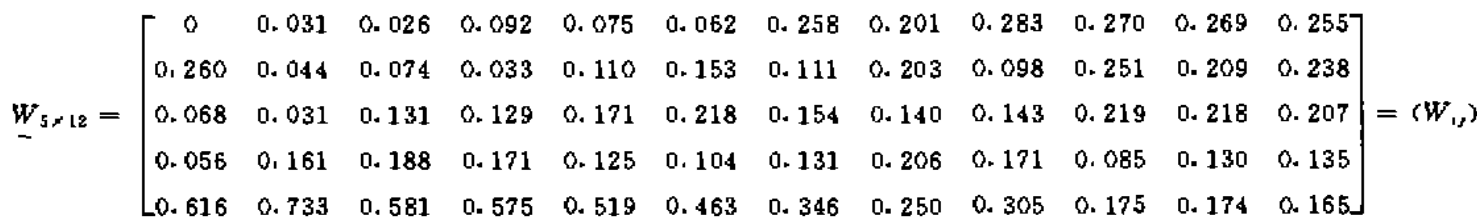

将矩阵 $\left(4^{\prime}\right),\left(6^{\prime}\right),\left(9^{\prime}\right)$ 中的有关数据, 代入公式 (18) 得到我国 12 个湖库对于各级营养 化隶凬度矩阵: 
按逮属度最大也考虑邻级逮属度较大原则，得评价结果见表3。表中也列出文[1]用水障 碍调查结果。由比较可见评价与调查实况相符。

表3 评价恉果与调查实况比较

Tab. 3 Comparison of the assessment results with the eutrophication observed

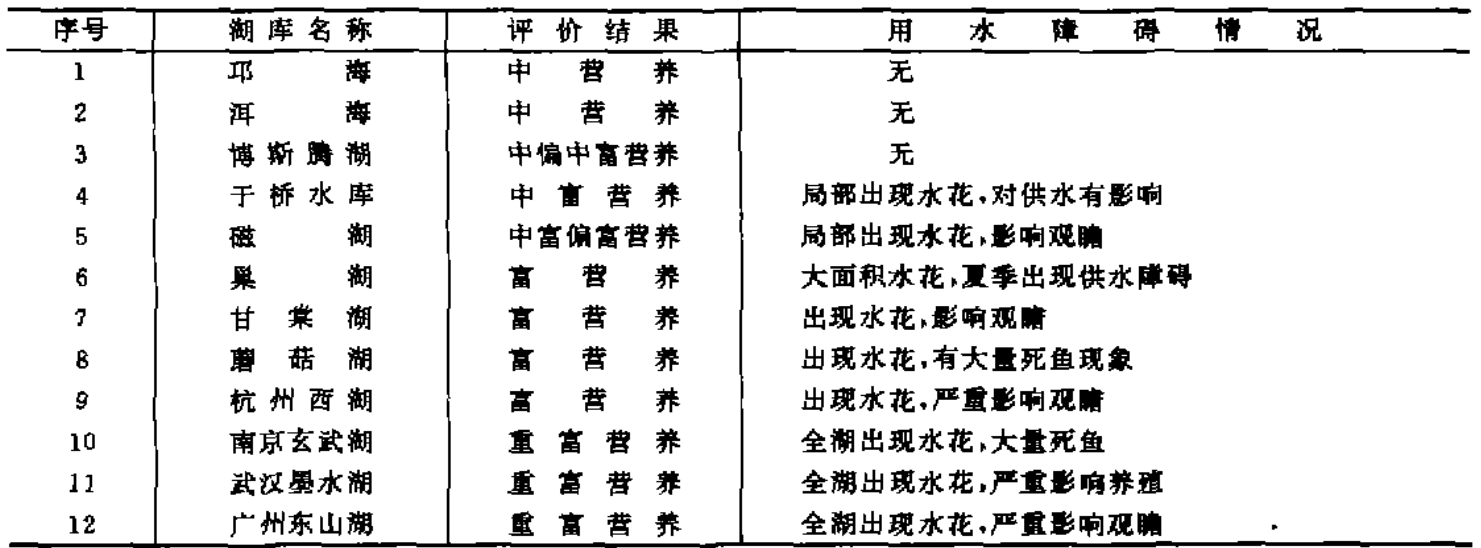

\section{六、结语}

本文提出的湖泊与水库富营养化模糊集评价模式基于“营养化”具有模糊性的论点，将 理论与实际结合起来。在评价中考虑了湖库样本(或测点)的超标权重与指标权重，提出的指 标综合权重模式与权重矩阵，比较贴切地反映了实际情况。建立的评价模式与指标综合权重 矩阵,原则上地可以在其它环境评价领域内应用。

\section{索文 姑}

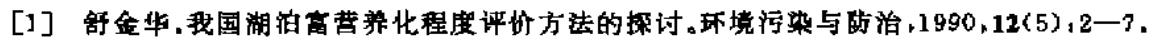

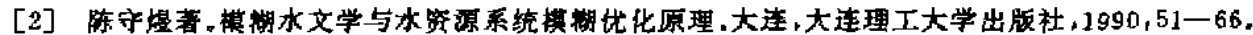

[3] 陈守煌、陈晓冰,水质模糊评价理㘮与模型。环境科学学报,1991,11《1),1一8.

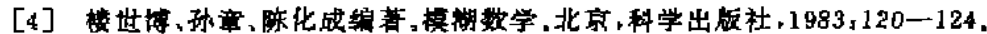




\title{
THE FUZZY SET THEORY AND MODEL OF ASSESSMENT FOR LAKE (RESERVOIR) EUTROPHICATION
}

\author{
Cheng Shouyu Xiong Deqi \\ (Datian Unutersity of Technology, Datran 116024)
}

\begin{abstract}
In this paper, the fuzzy set theory and model of assessment for lake (reservoir) eutrophication are established on the basis of the fuzzy characteristics of eutrophication. Furthermore, the paper proposed firstly the concept and derived method of comprehensive weight matrix of eutrophication indices. The model is also applied to assessing the eutrophication situations of 12 lakes (reservoirs) in China and the results are correspondent to the facts. Therefore, the theory and model provide not only scientific basis for the treatment and reservation of lake (reservoir) environment but also a new method for the study on assessment for lake (reservoir) eutrophication.

The theory and model proposed in the paper are also adaptable to other environmental fields concerning assessment theoretically.
\end{abstract}

Key words Fuzzy, assessment, lake (reservoir) eutrophication 